\section{ÜBRIGENS}

... steht die gängige Empfehlung, die Natrium und Kochsalzzufuhr bei Herzinsuffizienz zu begrenzen, auf wackeligen Beinen. US-Mediziner kamen jüngst in einer Studie zu dem Ergebnis, dass eine Natriumrestriktion mit einer Zunahme von durch Herzinsuffizienz bedingten Klinikeinweisungen assoziiert war.

... sind die klinischen Effekte einer Lipidsenkung mit Statinen von bemerkenswerter Dauerhaftigkeit. Das belegt eine neue Langzeit-Analyse von Daten der WOSCOPSStudie, deren Hauptergebnisse bereits 1995 publiziert worden sind. Demnach waren auch nach 20 Jahren die klinischen Vorteile einer auf wenige Jahre befristeten Cholesterinsenkung mit Pravastatin noch deutlich zu erkennen.

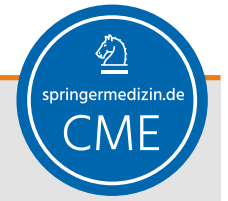

Punkte sammeln in der Sommerakademie mit e.Med

Auch 2016 haben wir in der Sommerakademie ausgewählte CME-Kurse für Ihre hausärztliche Fortbildung zusammengestellt.

Zum Thema "Kardiologie" finden Sie unter www.springermedizin.de/sommerakademie aktuell den Kurs

\section{„Update periphere arterielle}

\section{Verschlusskrankheit"}

Zur Teilnahme benötigen Sie das e.Med-Abo, mit dem Sie Zugang zu allen Inhalten auf Springer-Medizin.de erhalten - kombiniert mit einer gedruckten Fachzeitschrift Ihrer Wahl. Als e.Med-Abonnent haben Sie nicht nur Zugriff auf die Sommerakademie, Sie können das komplette CME-Kursangebot von Springer-Medizin.de nutzen. Hier finden Sie aktuell rund $550 \mathrm{CME}$-zertifizierte Fortbildungskurse aus allen medizinischen Fachrichtungen! Damit ist CME. SpringerMedizin.de Deutschlands erste Adresse für Online-CME-Fortbildung.

Nutzen Sie die Sommerzeit, um CME-Punkte zu sammeln und um sich schnell und effizient fortzubilden. Testen Sie CME.SpringerMedizin.de 30 Tage lang kostenlos und unverbindlich mit dem e.Med-Abo: www.springermedizin.de/eMed

SPRINT entfacht Diskussion

\title{
Blutdruck-Zielwerte wieder Thema
}

Eine der am meisten diskutierten Studien aus jüngster Zeit ist SPRINT. Sie hat die Diskussion über Blutdruck-Zielwerte erneut entfacht.

Hypertoniker mit hohem kardiovaskulären Risiko waren in der SPRINT-Studie mit Antihypertensiva entweder auf das Ziel eines systolischen Blutdrucks $<120 \mathrm{mmHg}$ oder das Standard-Ziel < $140 \mathrm{mmHg}$ eingestellt worden. De facto wurde in der intensiver behandelten Gruppe im Schnitt ein systolischer Wert von 121,4 mmHg und in der standardmäßig behandelten Gruppe ein Wert von 136,2 $\mathrm{mmHg}$ erreicht.

Das Risiko für den primären Endpunkt (Myokardinfarkt, akutes Koronarsyndrom, akute Herzinsuffizienz, kardiovaskulärer Tod) wurde durch die stärkere Blutdrucksenkung im Vergleich signifikant um 25\% und die Gesamtmortalität signifikant um 27\% reduziert. Das Herzinsuffizienz-Risiko verringerte sich relativ um $38 \%$.

Noch ist unklar, in welche konkrete Therapieempfehlungen künftiger HypertonieLeitlinien die Ergebnisse dieser Studien transformiert werden. Schon jetzt ist jedoch absehbar, dass die Studie keinen grundlegenden, alle Patienten mit Bluthochdruck gleichermaßen betreffenden Wandel in der Therapie herbeiführen wird.

Den SPRINT-Einschlusskriterien ist unschwer zu entnehmen, dass die Studie auf Hypertoniker mit hohem kardiovaskulärem Risiko fokussiert war. Damit scheint die Zielgruppe für eine intensivere Blutdrucksenkung klar umrissen zu sein. Patienten mit Diabetes, die einem beim Stichwort Hochrisikopatient sofort einfallen, waren allerdings von der Studie ebenso ausgeschlossen wie Patienten mit Schlaganfall oder Herzinsuffizienz in der Vorgeschichte. Einen niedriger angesetzten Universal-Zielwert für alle Hypertoniker wird es also nicht geben.

\section{Zunahme unerwünschter Effekte}

Die stärkere Senkung des Blutdrucks hatte ihren Preis. Unter dieser Therapie war in SPRINT nicht überraschend eine Zunahme unerwünschter Effekte wie Hypotonien, Synkopen und Elektrolytstörungen zu verzeichnen. Auch die $\mathrm{Zu-}$ nahme ungünstiger Effekte auf die Nierenfunktion könnte die Sorge verstärken, dass die intensivere Blutdrucksenkung speziell bei älteren Patienten mit Bluthochdruck vermehrt Probleme bereiten könnte.

Diesen Bedenken treten die SPRINTAutoren mit einer kürzlich veröffentlichen Substudie entgegen [1]. Danach haben die Vorteile der strikteren Blutdruckeinstellung auch bei Patienten im Alter über 75 Jahre Bestand. Auch in dieser Subgruppe reduzierte die ambitioniertere Therapie das Risiko für den primären kombinierten Endpunkt um 34\% und die Gesamtsterberate um 33\%.

1. JAMA 2016, online 19. Mai
Überraschende Studie

\section{Ernährung: Wie herzgesund ist Pflanzenöl?}

Fördert der Ersatz von gesättigten Fettsäuren etwa in tierischen Produkten durch Pflanzenöle mit einem hohen Gehalt an ungesättigten Fettsäuren die Herzgesundheit? Aber ja, werden viele antworten. Zweifel an dieser These wecken allerdings Ergebnisse einer bereits vor Jahrzehnten in Minnesota durchgeführten und kürzlich neue analysierten Studie bei mehr als 2300 Probanden [BMJ 2016; 353: i1246]. Zwar führte der Ersatz von gesättigten Fettsäuren durch vermehrten Konsum von Planzenölen mit hohem Linolsäure-Anteil zu einem Abfall der LDL-Cholesterinwerte. Eine Reduktion von kardiovaskulären Ereignissen hatte diese Ernährungsumstellung jedoch nicht zur Folge im Gegenteil: Bei der Mortalitätsrate zeigte sich ein unerwarteter Anstieg. 